OPEN ACCESS

Edited by:

Zlatko Kopecki,

University of South Australia, Australia

Reviewed by:

Anneliese Sophie Ashhurst The University of Sydney, Australia

Gil-Saeng Jeong,

Keimyung University, South Korea

*Correspondence: Jin Tae Hong jinthong@chungbuk.ac.kr Min Jong Song

bitsugar@catholic.ac.kr

Specialty section:

This article was submitted to Inflammation,

a section of the journal

Frontiers in Immunology

Received: 24 May 2020 Accepted: 04 November 2020 Published: 01 December 2020

Citation:

Lee YS, Jeon SH, Ham HJ, Lee HP,

Song MJ and Hong JT (2020) Improved Anti-Inflammatory

Effects of Liposomal Astaxanthin on a

Phthalic Anhydride-Induced Atopic Dermatitis Model.

Front. Immunol. 11:565285. doi: 10.3389/fimmu.2020.565285

\section{Improved Anti-Inflammatory Effects of Liposomal Astaxanthin on a Phthalic Anhydride-Induced Atopic Dermatitis Model}

\author{
Yong Sun Lee ${ }^{1}$, Seong Hee Jeon ${ }^{1}$, Hyeon Joo Ham ${ }^{1}$, Hee Pom Lee ${ }^{1}$, Min Jong Song ${ }^{2 \star}$ \\ and Jin Tae Hong ${ }^{1 *}$ \\ ${ }^{1}$ College of Pharmacy and Medical Research Center, Chungbuk National University, Cheongju, Chungbuk, South Korea, \\ 2 Department of Obstetrics and Gynecology, Yeouido St. Mary's Hospital, College of Medicine, The Catholic University of \\ Korea, Seoul, South Korea
}

Previously, we found that astaxanthin (AST) elicited an anti-inflammatory response in an experimental atopic dermatitis (AD) model. However, the use of AST was limited because of low bioavailability and solubility. We hypothesized that liposome formulation of AST could improve this. In this study, we compared the anti-inflammatory and anti-dermatotic effects of liposomal AST (L-AST) and free AST. We evaluated the effect of L-AST on a phthalic anhydride (PA)-induced animal model of $A D$ by analyzing morphological and histopathological changes. We measured the mRNA levels of AD-related cytokines in skin tissue and immunoglobulin E concentrations in the serum. Oxidative stress and transcriptional activities of signal transducer and activator of transcription 3 (STAT3) and nuclear factor (NF)$\kappa \mathrm{B}$ were analyzed via western blotting and enzyme-linked immunosorbent assay. PAinduced dermatitis severity, epidermal thickening, and infiltration of mast cells in skin tissues were ameliorated by L-AST treatment. L-AST suppressed AD-related inflammatory mediators and the inflammation markers, inducible nitric oxide synthase (iNOS) and cyclooxygenase (COX)-2 in PA-induced skin conditions. Oxidative stress and expression of antioxidant proteins, glutathione peroxidase-1 (GPx-1) and heme oxygenase-1 ( $\mathrm{HO}-1)$, were recovered by L-AST treatment in skin tissues from PA-induced mice. L-AST treatment reduced transcriptional activity of STAT3 and NF- $\mathrm{KB}$ in PA-induced skin tissues. Our results indicate that L-AST could be more effective than free AST for AD therapy.

Keywords: astaxanthin, liposome, atopic dermatitis, oxidative stress, signal transducer and activator of transcription 3, nuclear factor- $\mathrm{kB}$

\section{INTRODUCTION}

Atopic dermatitis $(\mathrm{AD})$ is a common chronic inflammatory skin disease characterized by mass release of cytokines (1). Stimulated keratinocytes release cytokines and chemokines associated with innate immunity, such as thymic stromal lymphopoietin (TSLP), interleukin (IL)-1 $\beta$, IL-33, chemokine (C-C motif) ligand (CCL)17, and CCL22 (2). Released mediators have been described to attract macrophages, type $2 \mathrm{~T}$ helper cells, and group 2 innate lymphoid cells, which secrete IL-4 
and IL-13 to induce immunoglobulin $\mathrm{E}(\operatorname{IgE})$ production $(3,4)$. Mast cells also contribute to $\mathrm{AD}$ development and $\operatorname{IgE}$ leads to the activation of mast cells through the release of cytokines into skin lesions (5). Thus, the reduction of these cytokines could be an important approach for AD therapy.

The skin is the largest organ of the human body, protecting it from harmful environmental factors, such as chemicals, biologic materials, and allergens. These external materials are known to cause oxidative stress by inducing the generation of reactive species in keratinocytes (6). Inflammatory responses are triggered by oxidative stress through the upregulation of proinflammatory cytokines (7). A previous study showed that TSLP, which is a trigger factor for the pathogenesis of $A D$, was increased by reactive oxygen species (ROS) in AD tissues (8). A clinical study indicated that the levels of various antioxidants, such as superoxide dismutase (SOD), glutathione peroxidase $(\mathrm{GPx})$, and glutathione (GSH), were substantially decreased in patients with AD compared to healthy controls (9). Thus, increased oxidative stress could contribute to the development of $\mathrm{AD}$.

Signal transducer and activator of transcription 3 (STAT3) and nuclear factor (NF)- $\mathrm{KB}$ are critical transcription factors that regulate inflammatory responses; they activate inflammationrelated genes, such as cyclooxygenase (COX)-2, tumor necrosis factor (TNF)- $\alpha$, and IL-6 (10). It is noteworthy that STAT3 is critical for regulating IgE levels and IgE-based allergen sensitization, as well as mast cell degranulation to release cytokines $(11,12)$. Thus, targeting STAT3 and NF- $\kappa \mathrm{B}$ could be a useful approach for AD treatment. The NF- $\kappa \mathrm{B}$ inhibitor IMD0354 alleviated experimental $\mathrm{AD}$ in $\mathrm{NC} / \mathrm{Nga}$ mice through the inhibition of $\mathrm{AD}$-related cytokines and infiltration of inflammatory cells (13). Topical NF- $\kappa B$ decoy oligonucleotides were shown to block chronic AD-like skin inflammation by the downregulation of Th1 and Th2 cytokines (14). Topical application of Momelotinib, a novel Janus kinase (JAK)1/JAK2 inhibitor, suppressed STAT3 signaling, the release of proinflammatory cytokines, total serum IgE levels, and mast cell infiltration, and thus improved the symptoms of AD (15).

Astaxanthin (3,3'-dihydroxy- $\beta, \beta$-carotene-4,4'-dione; AST) is a xanthophyll carotenoid usually found in microalgae and crustaceans, such as krill and shrimp (16). Previous studies have shown that AST is pharmacologically effective against various diseases, including cardiovascular, gastrointestinal, liver, neurodegenerative, and skin diseases, via its anti-oxidant and anti-inflammatory activities $(17,18)$. Our previous reports revealed that AST inhibited oxidative stress and inflammation via inactivation of STAT3 and NF- $\mathrm{KB}$ in ethanol-induced liver injury and lipopolysaccharide-induced neuroinflammation (19, 20). Topical application has been shown to provide advantages by reducing side effects, drug abuse and toxicity, allowing for high-dose application, while being easy to use, and avoiding firstpass metabolism $(21,22)$. Moreover, the topical route is wellsuited for sustained and controlled delivery over a prolonged period (21). With respect to skin diseases, topical application directly at the site of skin inflammation is indicated. However, astaxanthin has poor water-solubility, thus limiting direct application to the skin $(23,24)$. To solve this problem, liposomal formulation improves its solubility by conjugation with phospholipid structures (25).

In this study, we investigated the effect of liposomal AST (LAST) on the prevention of AD via inhibition of skin inflammation.

\section{MATERIALS AND METHODS}

\section{Preparation of Liposomal Astaxanthin}

AST, purchased from GDE Co., Ltd. (Siheung, Korea), was mixed with $70 \%$ ethanol at room temperature $\left(21-25^{\circ} \mathrm{C}\right)$, and insoluble materials were removed using Whatman filter paper. Phosphatidylcholine (from Soy) was dissolved in 95\% ethanol, and insoluble materials were removed using Whatman filter paper. AST and phosphatidylcholine were mixed at a 1:4 ratio using a high-pressure homogenizer (Microfluidizer ${ }^{\mathrm{TM}}$, cooling temperature: $-15 \sim-20^{\circ} \mathrm{C} / 1,000$ bar $/ 3$ cycles; 11-6094A000, Microfluidics Corp., MA, USA). After mixing, ethanol was removed via vacuum distillation. The solution was transferred to a $5 \mathrm{~mm}$ stainless steel plate after which all liquid components were removed using a vacuum freeze dryer. The solids from which the liquid components had been removed were collected and stored at room temperature to prepare L-AST for use in the experiment. The particle size of the L-AST produced was on average $64.5 \pm 2.8 \mathrm{~nm}$, which was determined using a particle size analyzer (ELS-Z, Otsuka electronics, Osaka, Japan).

\section{Animal Housing and Ethical Approval}

Animal experiments were performed in accordance with the guidelines for animal experiments of the Institutional Animal Care and Use Committee (IACUC) of the Laboratory Animal Research Center at Chungbuk National University, Korea. The experimental protocol was approved by the IACUC of the Laboratory Animal Research Center at Chungbuk National University, Korea (Ethical approval No. CBNUA-1304-19-01). Male SKH-1 mice (also known as HR-1 hairless mice) were obtained from Daehan Bio Link Co., Ltd. (Eumsung, Korea) and housed at controlled temperature $\left(21-25^{\circ} \mathrm{C}\right)$, relative humidity (55 $\pm 10 \%$ ), and $12 \mathrm{~h}$ light-dark cycles. Food and purified tap water were provided ad libitum.

\section{Phthalic Anhydride (PA)-Induced AD Development}

SKH-1 mice (8-week-old males) were randomly divided to five groups. PA solution $(5 \%, 200 \mu \mathrm{l})$ was applied on the dorsal skin three times a week for 4 weeks. AST $(1 \mathrm{mg} / \mathrm{ml} ; 200 \mu \mathrm{l})$ or L-AST $(0.5$ and $1 \mathrm{mg} / \mathrm{ml} ; 200 \mu \mathrm{l})$ was applied $3 \mathrm{~h}$ after every PA treatment. Vehicle-treated mice were used as a control. PA, AST, and L-AST were dissolved in acetone:olive oil (AOO) solution (4:1 ratio, v/v). The clinical score for each group was determined as none (0), mild (1), moderate (2), or severe (3) according to the average score of each symptom, i.e., erythema (redness), scaling, and itching, for each mouse. 


\section{Measurement of Body and Lymph Node Weight}

During the experimental period, the body weight of mice was measured once a week over the course of 4 weeks using an electronic scale (Mettler Toledo, Greifensee, Switzerland). Skin draining lymph nodes were collected and weighed using a precision electronic balance (FX-200i; A\&D Korea, Seoul, Korea).

\section{Immunohistochemistry (IHC)}

IHC was conducted as described previously (26). The slides were stained with specific primary antibodies. Mast cells were stained using toluidine blue solution (IHC world, Ellicott City, MD, USA). The average of epidermal thickness and the number of mast cells was calculated by a single measurement of six different fields (non-overlapping) in each group. Information on the antibodies used is provided in Supplementary Table S1.

\section{Western Blot Analysis}

Western blot analysis was performed as previously described (26). The membrane was incubated with specific primary antibodies directed against the following proteins: p50, GPx-1, heme oxygenase (HO)-1, STAT3, histone $\mathrm{H} 1$ and $\beta$-actin (Santa Cruz Biotechnology, CA, USA), p65, inducible nitric oxide synthase (iNOS) and COX-2 (Abcam, Cambridge, MA, USA), and $\mathrm{p}-\mathrm{I} \kappa \mathrm{B} \alpha, \mathrm{I} \kappa \mathrm{B} \alpha$ and $\mathrm{p}$-STAT3 (Cell Signaling, Beverly, MA, USA). Histone $\mathrm{H} 1$ and $\beta$-actin were used as loading controls. Band intensities were measured using the Fusion FX7 image acquisition system (Vilber Lourmat, Eberhardzell, Germany). Western blot band intensities were quantified using ImageJ software (NIH; Bethesda, MD, USA). Information on the antibodies used is provided in Supplementary Table S1.

\section{Quantitative Real-Time PCR (RT-qPCR)}

RT-qPCR was performed as described previously (26). Briefly, total RNA was collected from mouse skin tissues or lymph nodes using the Ribo ${ }^{\mathrm{EX}}$ RNA Extraction Kit (GeneAll Biotechnology, Seoul, Korea) and cDNA was synthesized using the High-Capacity RNAto-cDNA kit (Applied Biosystems, Foster City, CA, USA). RT-qPCR was performed using specific primers with the StepOnePlus ${ }^{\mathrm{TM}} \mathrm{PCR}$ System (Applied Biosystems, Foster City, CA, USA). Levels of mRNA were normalized to the $18 \mathrm{~S}$ sequence, which was used as a house-keeping control. The fold change between groups was determined for all targets using the $2^{\Delta \Delta \mathrm{Ct}}$ method. Specific primer sequences are described in Supplementary Table S2.

\section{Serum IgE Assay}

Blood was collected from sacrificed mice and serum was isolated via centrifugation $\left(3,500 \mathrm{rpm}\right.$ for $7 \mathrm{~min}$ at $\left.4^{\circ} \mathrm{C}\right)$ using blood collection tube (BD Microtainer ${ }^{\circledR}$, Franklin Lakes, NJ, USA). Serum levels of mouse IgE were measured using the ELISA kit of KOMA Biotech (Seoul, Korea) according to the manufacturer's protocol.

\section{Oxidative Stress Assay}

Hydrogen peroxide $\left(\mathrm{H}_{2} \mathrm{O}_{2}\right)$ was measured using the Hydrogen Peroxide Assay Kit (Biovision, Milpitas, CA, USA). The levels of GSH and oxidized glutathione (GSSG) were analyzed using the
GSH/GSSG Ratio Detection Assay Kit (Abcam, Cambridge, MA, USA). Malondialdehyde (MDA) levels were measured using TBARS Assay Kit according to the manufacturer's instructions (Cayman, Ann Arbor, MI, USA).

\section{Statistical Analysis}

All experiments were repeated at least three times to ensure reproducibility of the results. Statistical analysis was performed with GraphPad Prism 4.03 software (San Diego, CA, USA). Group differences were analyzed by one-way analysis of variance followed by Tukey's multiple comparison test. All values are presented as the mean \pm SD. Significance was set at a $\mathrm{p}<0.05$ for all tests. ${ }^{*}$ Control vs. PA; ${ }^{\dagger} \mathrm{PA}$ vs. PA + AST1, PA + L-AST0.5, and PA + LAST1; ${ }^{*} \mathrm{PA}+\mathrm{AST} 1$ vs. PA + L-AST0.5 and PA + L-AST1.

\section{RESULTS}

\section{Effects of L-AST Treatment on Experimental AD Development}

PA exposure is known to cause irritation, potentially leading to allergic skin diseases, such as contact dermatitis and $\mathrm{AD}$ by inducing allergyrelated cytokines, chemokines, and $\operatorname{IgE}(27,28)$. To investigate whether L-AST had an improved potential to prevent AD compared to AST, we used a PA-induced AD model. As shown in Figure 1A, we induced experimental AD. First, we measured the changes in body weight over the course of the experimental period. No substantial body weight changes were detected upon either AST or LAST treatment (Figure 1B). Next, we compared the associated AD symptoms, consisting of erythema (redness), scaling, and itching, for each group. It was revealed that $\mathrm{AD}$ symptoms and clinical scores were increased in the PA-induced group (Figures 1C, D). However, these symptoms and scores were significantly reduced upon AST treatment (Figures 1C, D). L-AST treatment further reduced PA-induced AD development in mice when compared with AST-treated mice (Figures 1C, D). As shown in Figure 1D, AST dyed the skin surface red, whereas L-AST did not color the skin. Western blot analysis showed that the reduction in the expression of the inflammation markers COX-2 and $\mathrm{NNOS}$ was more pronounced in L-AST treated mice when compared to AST treated mice (Figure 1E).

\section{Effect of L-AST Treatment on AD-Like Skin Inflammation}

Histological analysis of the skin tissues showed that AST and L-AST treatment reduced epidermal thickening in PA-induced skin tissues, with L-AST being more effective than free AST (Figure 2A). The reduction in PA-induced mast cell infiltration was also more pronounced in L-AST-treated mice than in AST-treated mice (Figure 2A). We further investigated the weight of skin draining lymph nodes, which serve as an indicator of skin inflammation. The result showed that AST and L-AST moderately and sufficiently reduced the PA-induced increase in lymph node weight and length (major axis), respectively (Figure 2B). Various inflammatory cytokines and chemokines are critical factors for $\mathrm{AD}$ development (2). Therefore, we analyzed AD-related mediators of PA-induced skin conditions. RT-qPCR analysis showed that the 


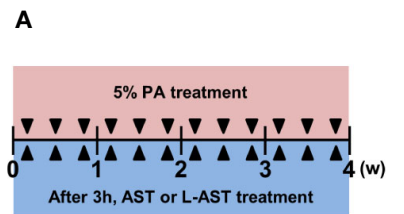

D
B

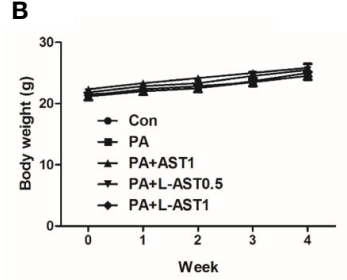

C

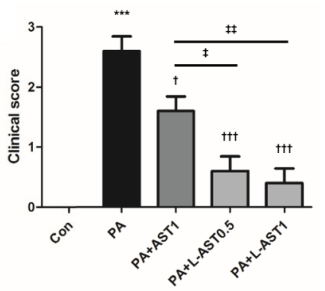

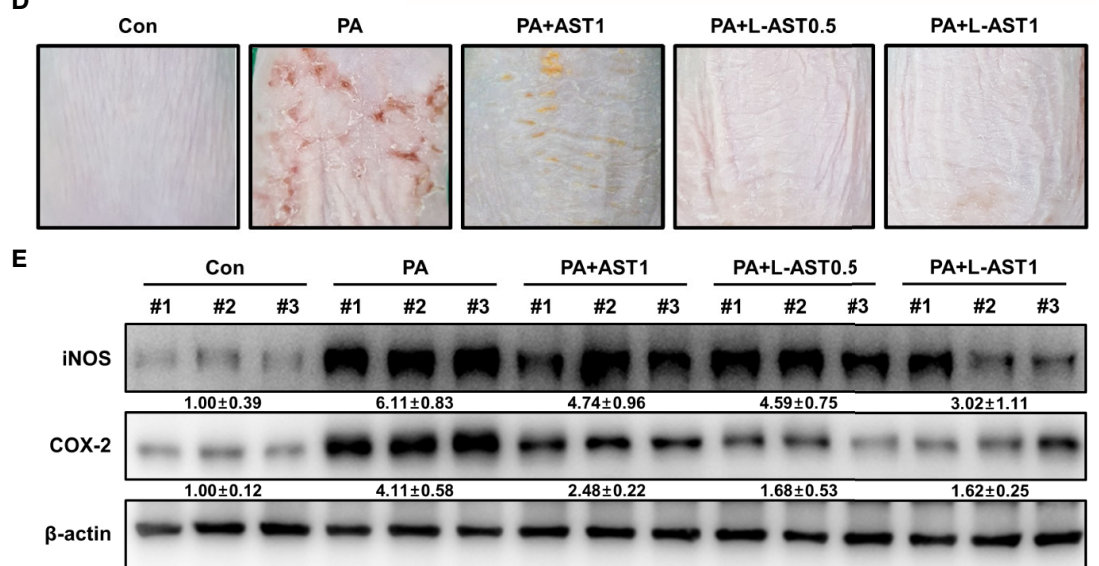

FIGURE 1 | Liposomal astaxanthin inhibits PA-induced atopic dermatitis. (A) Experimental schedule of PA-induced atopic dermatitis model. Mice were treated with 5\% PA solution three times a week for 4 weeks. AST or L-AST were applied $3 \mathrm{~h}$ after every PA treatment. (B) Body weight changes during 4-week experiment. (C, D) The morphological changes in mice after 4-week treatment as described in the Materials and Methods. Bar graphs indicate clinical score (C). $n=6$. The photographs are representative of each group of mice (D). (E) Expression of inflammation markers, COX-2 and iNOS, in skin tissues of each group. \#1-3 refer to skin tissues of the different mice in the same group. ${ }^{\dagger, \ddagger} p<0.05,{ }^{\ddagger \ddagger} p<0.01$ and ${ }^{* \star \star},{ }^{\dagger \dagger \dagger} p<0.001$.

A

Con

PA

PA+AST1

PA+L-AST0.5 PA+L-AST1
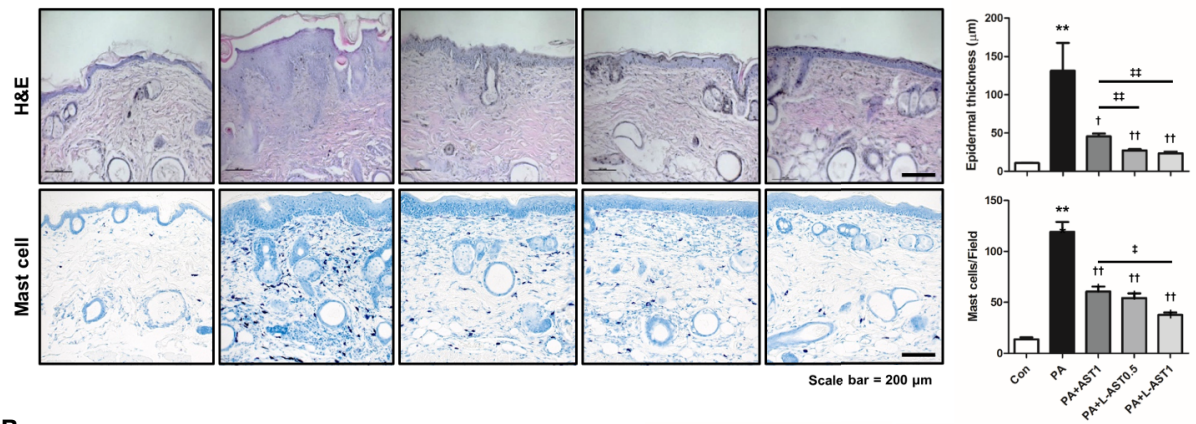

B
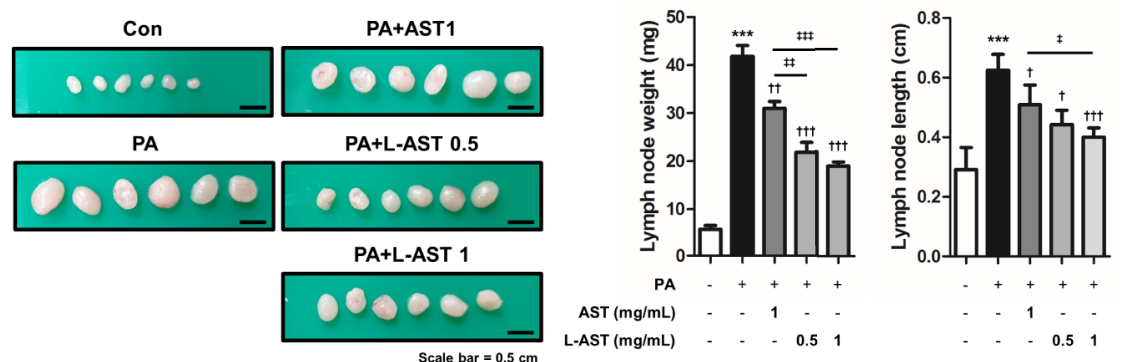

FIGURE 2 | Liposomal astaxanthin reduces epidermal thickening, lymph node weight, and expression of inflammatory markers. (A) Histological changes in mice after 4-week PA treatment. Bar graphs indicate epidermal thickness and mast cell number. $n=6$. (B) Changes of skin draining lymph node weight and length (major

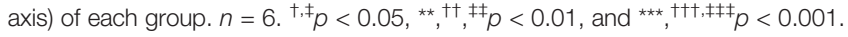


expression of Th2-related cytokines and chemokines, including TSLP, IL-4, IL-5, IL-13, IL-31, IL-33, CCL17, and CCL22, was increased in PA-induced skin tissues, but treatment with AST reduced the expression of these cytokines (Figure 3A). Furthermore, L-AST suppressed Th2-related mediators more effectively (Figure 3A). The increase in PA-induced Th1 cytokines, such as TNF- $\alpha$, IL- $1 \beta$, and IL- 6 , was markedly reduced in skin tissues of L-AST-treated mice compared to AST-treated mice (Figure 3B). Next, we evaluated serum levels of IgE, an indicator of allergic inflammation. Serum IgE was increased in PA-treated mice; these PA-induced serum IgE levels were significantly reduced in AST-treated mice (Figure 3C). L-AST was more efficient in decreasing serum IgE levels than free AST (Figure 3C). Further analysis indicated that L-AST treatment reduced AD-related cytokine levels (IL-1 $\beta$, IL-6, IL-4, and IL-13) in lymph nodes to a greater extent than AST treatment (Supplementary Figure S1).

\section{Effect of L-AST Treatment on Oxidative Stress}

Previous studies have shown that oxidative stress is related to $\mathrm{AD}(7$, 9). Furthermore, AST possesses antioxidant properties (29). Therefore, we evaluated oxidative stress in PA-induced skin conditions. The level of MDA, a marker of lipid peroxidation, was elevated by PA treatment, but reduced by the application of free AST and L-AST (Figure 4A). Additionally, PA-induced $\mathrm{H}_{2} \mathrm{O}_{2}$ levels were alleviated by free AST and L-AST treatments (Figure 4A). In addition, decreased GSH levels and GSH/GSSG ratios were recovered by the treatment of AST and L-AST (Figure 4A). Treatment with the latter resulted in enhanced suppression of oxidative stress compared to AST treatment (Figure 4A). We further investigated the expression of antioxidant-related genes, such as GPx-1 and HO-1. Western blot analysis showed that reduced expression of HO-1 and GPx-1 was recovered by AST in PA-induced mice (Figure 4B). L-AST was more effective in reversing GPx-1 and HO-1 expression levels compared to free AST (Figure 4B).

\section{Effect of L-AST Treatment on STAT3 and NF-KB Signaling Activation}

STAT3 and NF- $\mathrm{KB}$ are both involved in various inflammatory diseases including $\mathrm{AD}$. Recent studies demonstrated that ROS induced the activation of STAT3 and NF- $\kappa B$ signaling $(30,31)$. To investigate whether L-AST treatment further inhibited STAT3 and $N F-\kappa B$ activation in a PA-induced AD model, we analyzed the activation of STAT3 and NF- $\mathrm{KB}$ using western blot and IHC analysis. PA-treated mice were characterized by an increase in the phosphorylation of I $\mathrm{B} \alpha \alpha$ and STAT3 in cytoplasmic fractions, and by nuclear translocation of $\mathrm{p} 50$ and p65, as revealed by comparing cytoplasmic and nuclear fractions (Figure 5A). In contrast, AST treatment inhibited STAT3 and NF- $\kappa B$ activation (Figure 5A). In addition, L-AST treatment suppressed the activation of STAT3 and NF- $\kappa$ B more efficiently than free AST (Figure 5A). Similar to western blot analysis, IHC showed that AST treatment reduced phosphorylation of STAT3 and p65 in skin tissues upon PA treatment (Figure 5B). Moreover, L-AST was more potent than
AST in inhibiting the activation of STAT3 and p65 in PA-induced skin conditions.

\section{DISCUSSION}

$\mathrm{AD}$ is a chronic inflammatory skin disease, which is characterized by increased IgE levels and inflammatory cytokine expression (32, 33). Targeting of AD-related inflammatory mediators and antiinflammatory compounds are promising approaches for $\mathrm{AD}$ therapy (34). Previous studies reported that green tea extracts from tannase digests inhibited skin inflammation and mast cell infiltration in house dust mite antigen-induced AD-like lesions (35). Ethanol extracts of Ampelopsis brevipedunculata rhizomes inhibited an AD-like skin inflammation through downregulation of serum IgE levels and expression of TNF- $\alpha$, interferon (IFN)- $\gamma$, IL-4, IL-13, and IL-31 in a BALB/c AD model (36). In a previous study, we also found that Centella asiatica reduced PA-induced AD through its anti-inflammatory effects (37). In line with these findings, in our present study, significantly reduced AD-related cytokine release was observed in the L-AST-treated group. These data indicate that L-AST constitutes a promising candidate for the development of a therapeutic agent for AD treatment.

Oxidative stress has been associated with inflammatory diseases (30). Accordingly, endogenous and environmental pro-oxidants could induce ROS, causing oxidative damage, such as DNA modifications, lipid peroxidation, and inflammatory responses (38). However, the relationship between oxidative stress and AD is not clear. Previous reports were in support of suppression of oxidative stress being capable of alleviating AD. N-acetyl-Lcysteine, a precursor of GSH, decreased the levels of IL-4, IL-5, and IFN- $\gamma$ in Th2 cells (39). A clinical study showed that MDA levels were increased, but antioxidant parameters, including SOD, $\mathrm{GSH}, \mathrm{GPx}$, and vitamins (A,C, and E), were significantly decreased in blood samples from patients with $\mathrm{AD}$ compared to healthy controls (9). It has been reported that urinary concentrations of pentosidine and 8-hydroxy-2'-deoxyguanosine were higher in patients with $\mathrm{AD}$ compared to the controls (40). Furthermore, our data revealed that treatment with free AST or L-AST could decrease the levels of MDA and $\mathrm{H}_{2} \mathrm{O}_{2}$, but resulted in an increased GSH/GSSG ratio. These factors implicated that inhibition of oxidative stress may contribute to preventing $\mathrm{AD}$.

STAT3 and NF- $\kappa$ B are transcriptional factors significantly contributing to the development of $\mathrm{AD}$ as they play important roles in the regulation of AD-related inflammatory mediators (14, 41). Several STAT3 inhibitors, such as momelotinib, a novel JAK1/ JAK2 inhibitor, and the JAK inhibitor JTE-052 downregulated the STAT3-specific signal, release of pro-inflammatory cytokines, total serum IgE levels, and mast cell numbers, and concomitantly improved the symptoms of $\mathrm{AD}(15,42)$. NF- $\mathrm{KB}$ inhibitors alleviated $\mathrm{AD}$-like skin inflammation by inhibiting of inflammatory regulators and the infiltration of immune cells $(13,14)$. Our results showed that L-AST inhibited phosphorylation of IкB $\alpha$ and STAT3, as well as nuclear translocation of p50 and p65 in PA-induced skin conditions. These data suggest that L-AST-mediated inhibition of STAT3 and NF- $\mathrm{KB}$ could be essential for its anti-AD effect. 

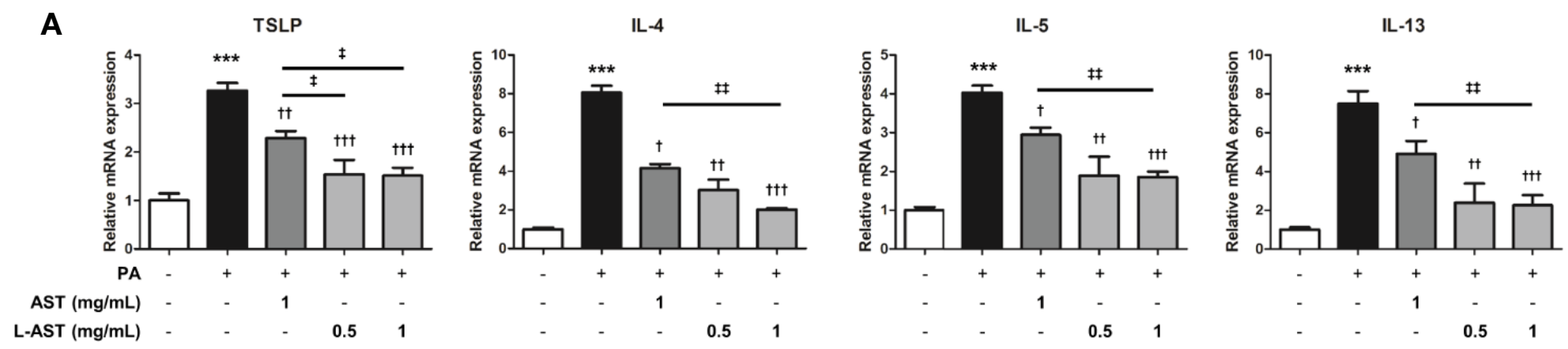

L-AST $(\mathrm{mg} / \mathrm{mL})$
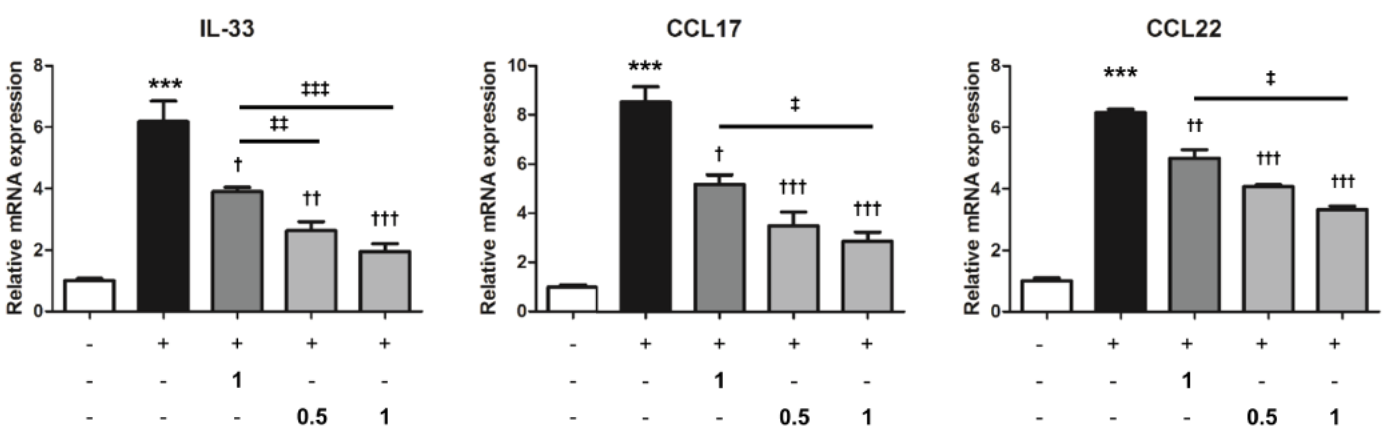

$$
\begin{array}{r}
\text { AST }(\mathrm{mg} / \mathrm{mL}) \\
\text { L-AST }(\mathrm{mg} / \mathrm{mL})
\end{array}
$$

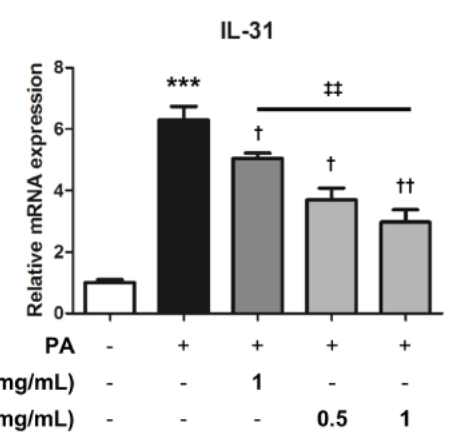

B
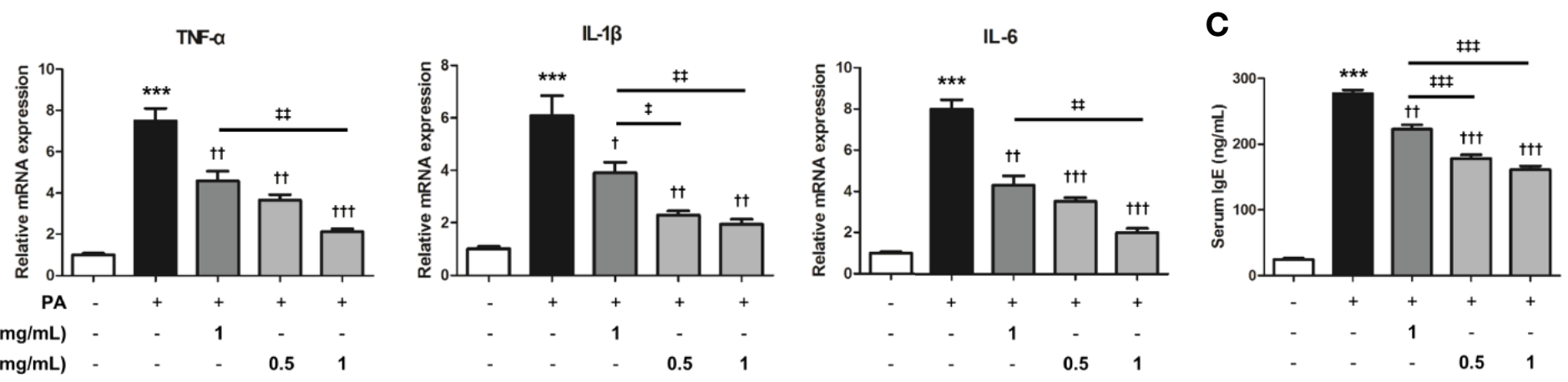

FIGURE 3 | Liposomal astaxanthin suppresses skin inflammation. (A) mRNA expression of Th2-related cytokines, TSLP, IL-4, IL-5, IL-13, IL-31, IL-33, CCL17, and CCL22 in skin tissues. $n=4$. (B) mRNA

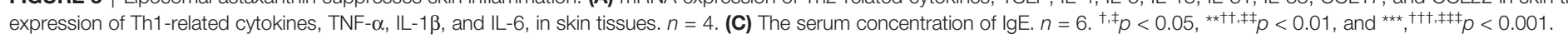




\section{A}

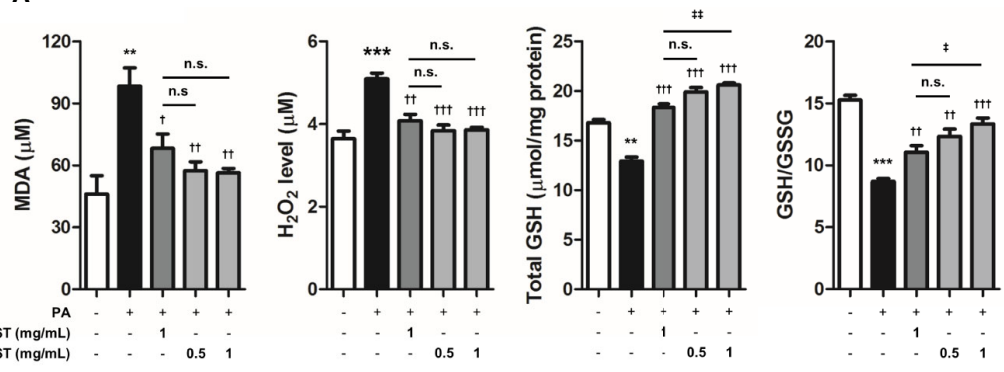

B

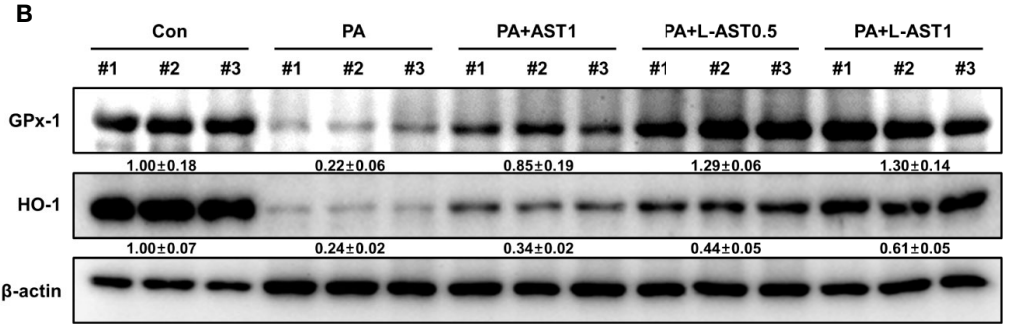

FIGURE 4 | Liposomal astaxanthin alleviates oxidative stress in PA-induced skin tissues. (A) MDA levels, $\mathrm{H}_{2} \mathrm{O}_{2}$ levels, total GSH, and GSH/GSSG ratio in skin tissues. $n=6$. (B) Expression of antioxidant-related markers, GPx-1, and HO-1, in skin tissues. \#1-3 refer to skin tissues of the different mice in the same group. ${ }^{\dagger, \pm} p<0.05,{ }^{* *,+\dagger, \neq \pm} p<0.01$, and ${ }^{* \star *,+\dagger+, \neq \neq \pm} p<0.001$.

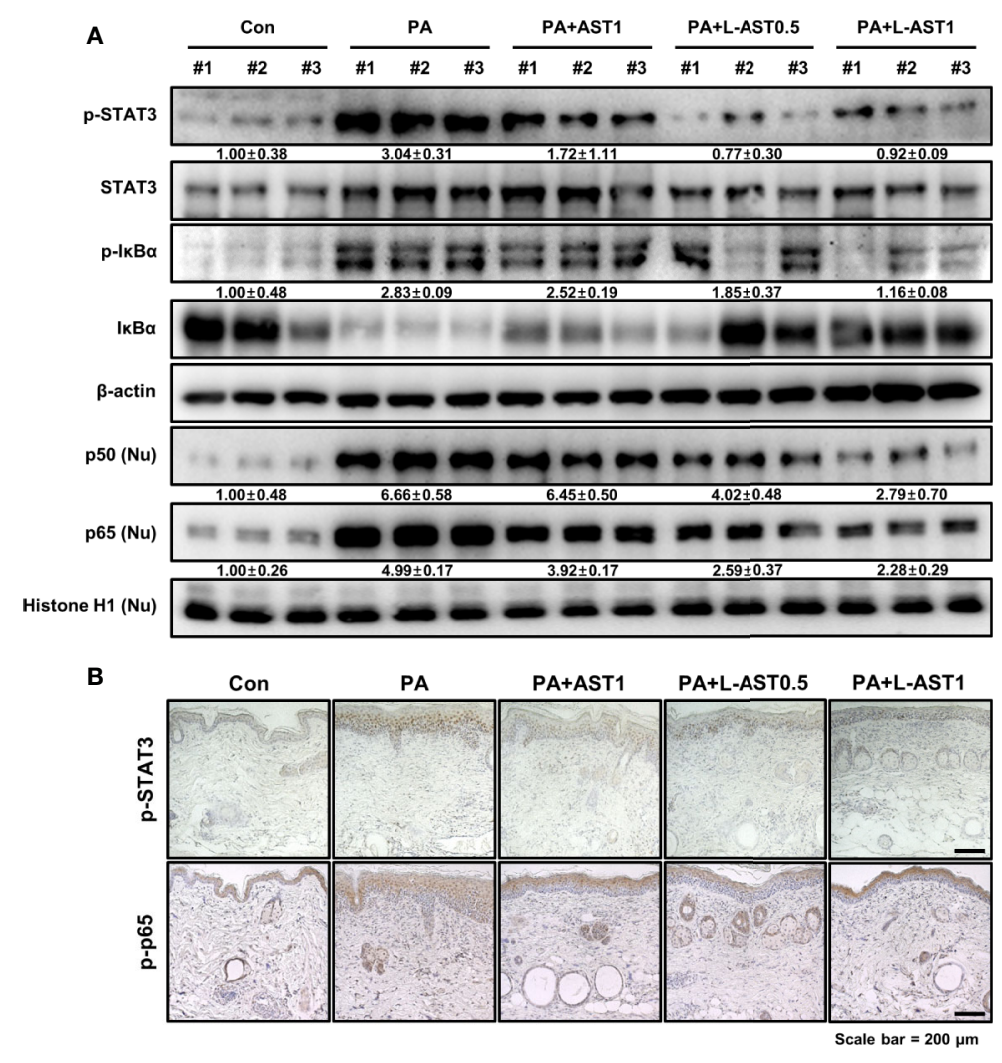

FIGURE 5 | Effect of liposomal astaxanthin on STAT3 and NF-kB signaling in PA-induced AD model. (A) Expression of phosphorylated STAT3 and IkB $\alpha$ in whole tissue lysates and nuclear translocation of p50 and p65 in nuclear fractions by Western blot analysis in skin tissues. \#1-3 refer to skin tissues of the different mice in the same group. (B) Representative IHC images showing phospho-STAT3 and phospho-p65 in PA-induced skin tissues. 
Topical administration has several advantages for treatment of skin diseases. Transdermal delivery can avoid metabolism of drugs by the liver, reduce side effects, and achieve local effects (43). Topical administration of AST effectively inhibited UVinduced ocular photokeratitis and AD-like skin inflammation $(44,45)$. Nevertheless, AST is both hydrophobic and hydrophilic and has poor water-solubility, rendering it unsuitable for skin application. To overcome this problem, various methods have been developed (46). Among them, liposome formulation is widely used for skin delivery systems as it is associated with increased drug solubilization (46). Additionally, liposome formulation could increase the transdermal delivery of AST. As demonstrated by many studies, liposome formulations are superior to the free forms with regard to skin delivery (47). A previous study indicated that liposomal adenosylcobalamin hydrogel improved skin permeation and reduced $\mathrm{AD}$ symptoms more efficiently than the non-liposomal type in a dichloronitrobenzene-induced $\mathrm{AD}$ mouse model (48). It has also been reported that liposomal betamethasone exerted stronger increased anti-inflammatory actions in patients with $\mathrm{AD}$ than in controls (49). It needs to be confirmed in future studies whether L-AST has increased skin permeability compared to AST.

This study demonstrated that L-AST treatment could prevent inflammatory cytokine release and oxidative stress in a PAinduced AD model more efficiently than free AST. Thus, liposomal formulation enhances the therapeutic efficacy of AST and has more practical applicability.

\section{DATA AVAILABILITY STATEMENT}

The original contributions presented in the study are included in the article/Supplementary Material. Further inquiries can be directed to the corresponding authors.

\section{REFERENCES}

1. Bieber T. Atopic dermatitis. Ann Dermatol (2010) 22(2):125-37. doi: 10.5021/ ad.2010.22.2.125

2. Weidinger S, Beck LA, Bieber T, Kabashima K, Irvine AD. Atopic dermatitis. Nat Rev Dis Primers (2018) 4(1):1. doi: 10.1038/s41572-018-0001-Z

3. Ito T, Wang Y-H, Duramad O, Hori T, Delespesse GJ, Watanabe N, et al. TSLP-activated dendritic cells induce an inflammatory $\mathrm{T}$ helper type 2 cell response through OX40 ligand. J Exp Med (2005) 202(9):1213-23. doi: 10.1084/jem.20051135

4. Doherty TA, Broide DH. Group 2 innate lymphoid cells: new players in human allergic diseases. J Invest Allergol Clin Immunol (2015) 25(1):1-11.

5. Kawakami T, Ando T, Kimura M, Wilson BS, Kawakami Y. Mast cells in atopic dermatitis. Curr Opin Immunol (2009) 21(6):666-78. doi: 10.1016/j.coi.2009.09.006

6. Corsini E, Galbiati V, Nikitovic D, Tsatsakis AM. Role of oxidative stress in chemical allergens induced skin cells activation. Food Chem Toxicol (2013) 61:74-81. doi: 10.1016/j.fct.2013.02.038

7. Ji H, Li X-K. Oxidative Stress in Atopic Dermatitis. Oxid Med Cell Longev (2016) 2016:8. doi: 10.1155/2016/2721469

8. Kim H-J, Lee E, Lee S-H, Kang M-J, Hong S-J. Mold elicits atopic dermatitis by reactive oxygen species: Epidemiology and mechanism studies. Clin Immunol (2015) 161(2):384-90. doi: 10.1016/j.clim.2015.07.007

9. Sivaranjani N, Rao SV, Rajeev G. Role of reactive oxygen species and antioxidants in atopic dermatitis. J Clin Diagn Res (2013) 7(12):2683-5. doi: $10.7860 /$ JCDR/2013/6635.3732

\section{ETHICS STATEMENT}

The animal study was reviewed and approved by Institutional Animal Care and Use Committee (IACUC) of Laboratory Animal Research Center at Chungbuk National University, Korea (Ethical approval No. CBNUA-1304-19-01).

\section{AUTHOR CONTRIBUTIONS}

YL conducted most of the experiments, performed data analyses, designed the experiments, and wrote the manuscript. SJ and $\mathrm{HH}$ assisted in animal experiments. HL provided experimental advices. MS and $\mathrm{JH}$ supervised the entire project and contributed profoundly to experimental design, data interpretation, and revision the manuscript. All authors contributed to the article and approved the submitted version.

\section{FUNDING}

This work is financially supported by the National Research Foundation of Korea [NRF] grant funded by the Korean government (MSIP) (No. MRC, 2017R1A5A2015541).

\section{SUPPLEMENTARY MATERIAL}

The Supplementary Material for this article can be found online at: https://www.frontiersin.org/articles/10.3389/fimmu.2020. 565285/full\#supplementary-material

10. Baumgart S, Ellenrieder V, Fernandez-Zapico ME. Oncogenic transcription factors: cornerstones of inflammation-linked pancreatic carcinogenesis. Gut (2013) 62(2):310-6. doi: 10.1136/gutjnl-2011-301008

11. Boos AC, Hagl B, Schlesinger A, Halm BE, Ballenberger N, Pinarci M, et al. Atopic dermatitis, STAT3- and DOCK8-hyper-IgE syndromes differ in IgE-based sensitization pattern. Allergy (2014) 69(7):943-53. doi: 10.1111/all.12416

12. Siegel AM, Stone KD, Cruse G, Lawrence MG, Olivera A, Jung MY, et al. Diminished allergic disease in patients with STAT3 mutations reveals a role for STAT3 signaling in mast cell degranulation. J Allergy Clin Immunol (2013) 132(6):1388-96. doi: 10.1016/j.jaci.2013.08.045

13. Tanaka A, Muto S, Jung K, Itai A, Matsuda H. Topical Application with a New

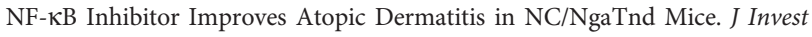
Dermatol (2007) 127(4):855-63. doi: 10.1038/sj.jid.5700603

14. Dajee M, Muchamuel T, Schryver B, Oo A, Alleman-Sposeto J, De Vry CG, et al. Blockade of Experimental Atopic Dermatitis via Topical NF- $\kappa B$ Decoy Oligonucleotide. J Invest Dermatol (2006) 126(8):1792-803. doi: 10.1038/sj.jid.5700307

15. Jin W, Huang W, Chen L, Jin M, Wang Q, Gao Z, et al. Topical Application of JAK1/JAK2 Inhibitor Momelotinib Exhibits Significant Anti-Inflammatory Responses in DNCB-Induced Atopic Dermatitis Model Mice. Int J Mol Sci (2018) 19(12):3973. doi: 10.3390/ijms19123973

16. Fassett RG, Coombes JS. Astaxanthin: a potential therapeutic agent in cardiovascular disease. Mar Drugs (2011) 9(3):447-65. doi: 10.3390/ md9030447

17. Yamashita E. Astaxanthin as a Medical Food. Funct Foods Health Dis (2013) 3 (7):254-8. doi: 10.31989/ffhd.v3i7.49 
18. Fakhri S, Abbaszadeh F, Dargahi L, Jorjani M. Astaxanthin: A mechanistic review on its biological activities and health benefits. Pharmacol Res (2018) 136:1-20. doi: 10.1016/j.phrs.2018.08.012

19. Han JH, Ju JH, Lee YS, Park JH, Yeo IJ, Park MH, et al. Astaxanthin alleviated ethanol-induced liver injury by inhibition of oxidative stress and inflammatory responses via blocking of STAT3 activity. Sci Rep (2018) 8(1):14090. doi: 10.1038/ s41598-018-32497-w

20. Han JH, Lee YS, Im JH, Ham YW, Lee HP, Han SB, et al. Astaxanthin Ameliorates Lipopolysaccharide-Induced Neuroinflammation, Oxidative Stress and Memory Dysfunction through Inactivation of the Signal Transducer and Activator of Transcription 3 Pathway. Mar Drugs (2019) 17(2):123. doi: 10.3390/md17020123

21. Brown MB, Martin GP, Jones SA, Akomeah FK. Dermal and Transdermal Drug Delivery Systems: Current and Future Prospects. Drug Delivery (2006) 13(3):175-87. doi: 10.1080/10717540500455975

22. Hagen M, Baker M. Skin penetration and tissue permeation after topical administration of diclofenac. Curr Med Res Opin (2017) 33(9):1623-34. doi: 10.1080/03007995.2017.1352497

23. Pan L, Wang H, Gu K. Nanoliposomes as Vehicles for Astaxanthin: Characterization, In Vitro Release Evaluation and Structure. Molecules (2018) 23(11):2822. doi: 10.3390/molecules23112822

24. Hama S, Takahashi K, Inai Y, Shiota K, Sakamoto R, Yamada A, et al. Protective Effects of Topical Application of a Poorly Soluble Antioxidant Astaxanthin Liposomal Formulation on Ultraviolet-Induced Skin Damage. J Pharma Sci (2012) 101(8):2909-16. doi: 10.1002/jps.23216

25. Peralta MF, Guzmán ML, Pérez AP, Apezteguia GA, Fórmica ML, Romero EL, et al. Liposomes can both enhance or reduce drugs penetration through the skin. Sci Rep (2018) 8(1):13253-3. doi: 10.1038/s41598-018-31693-y

26. Lee YS, Lee CH, Bae JT, Nam KT, Moon DB, Hwang OK, et al. Inhibition of skin carcinogenesis by suppression of NF- $\kappa$ B dependent ITGAV and TIMP-1 expression in IL-32 $\gamma$ overexpressed condition. J Exp Clin Cancer Res (2018) 37 (1):293. doi: 10.1186/s13046-018-0943-8

27. Bae CJ, Shim SB, Jee SW, Lee SH, Kim MR, Lee JW, et al. IL-6, VEGF, KC and RANTES are a major cause of a high irritant dermatitis to phthalic anhydride in C57BL/6 inbred mice. Allergol Int (2010) 59(4):389-97. doi: 10.2332/ allergolint.10-OA-0207

28. Mok JY, Jeon IH, Cho J-K, Park JM, Kim HS, Kang HJ, et al. Effect of Persimmon Leaf Extract on Phthalic Anhydride-induced Allergic Response in Mice. Prev Nutr Food Sci (2012) 17(1):14-21. doi: 10.3746/pnf.2012.17.1.014

29. Davinelli S, Nielsen ME, Scapagnini G. Astaxanthin in Skin Health, Repair, and Disease: A Comprehensive Review. Nutrients (2018) 10(4):522. doi: $10.3390 /$ nu10040522

30. Reuter S, Gupta SC, Chaturvedi MM, Aggarwal BB. Oxidative stress, inflammation, and cancer: how are they linked? Free Radic Biol Med (2010) 49(11):1603-16. doi: 10.1016/j.freeradbiomed.2010.09.006

31. Liu H, Liu X, Zhang C, Zhu H, Xu Q, Bu Y, et al. Redox Imbalance in the Development of Colorectal Cancer. J Cancer (2017) 8(9):1586-97. doi: 10.7150/jca.18735

32. Chen L, Martinez O, Overbergh L, Mathieu C, Prabhakar BS, Chan LS. Early up-regulation of Th2 cytokines and late surge of Th1 cytokines in an atopic dermatitis model. Clin Exp Immunol (2004) 138(3):375-87. doi: 10.1111/ j.1365-2249.2004.02649.x

33. Neis MM, Peters B, Dreuw A, Wenzel J, Bieber T, Mauch C, et al. Enhanced expression levels of IL-31 correlate with IL-4 and IL-13 in atopic and allergic contact dermatitis. J Allergy Clin Immunol (2006) 118(4):930-7. doi: 10.1016/j.jaci.2006.07.015

34. Heratizadeh A, Werfel T. Anti-inflammatory therapies in atopic dermatitis. Allergy (2016) 71(12):1666-75. doi: 10.1111/all.13065

35. Hwang Y, Chang B, Kim T, Kim S. Ameliorative effects of green tea extract from tannase digests on house dust mite antigen-induced atopic dermatitislike lesions in NC/Nga mice. Arch Dermatol Res (2019) 311(2):109-20. doi: 10.1007/s00403-018-01886-6
36. Choi Y-A, Yu J-H, Jung HD, Lee S, Park P-H, Lee H-S. et al: Inhibitory effect of ethanol extract of Ampelopsis brevipedunculata rhizomes on atopic dermatitis-like skin inflammation. J Ethnopharmacol (2019) 238:111850. doi: 10.1016/j.jep.2019.111850

37. Park JH, Choi JY, Son DJ, Park EK, Song MJ, Hellström M, et al. AntiInflammatory Effect of Titrated Extract of Centella asiatica in Phthalic Anhydride-Induced Allergic Dermatitis Animal Model. Int J Mol Sci (2017) 18(4):738. doi: 10.3390/ijms18040738

38. Briganti S, Picardo M. Antioxidant activity, lipid peroxidation and skin diseases. What's new. J Eur Acad Dermatol Venereol (2003) 17(6):663-9. doi: 10.1046/j.1468-3083.2003.00751.x

39. Bengtsson A, Lundberg M, Avila-Cariño J, Jacobsson G, Holmgren A, Scheynius A. Thiols decrease cytokine levels and down-regulate the expression of CD30 on human allergen-specific T helper (Th) 0 and Th2 cells. Clin Exp Immunol (2001) 123(3):350-60. doi: 10.1046/j.1365-2249.2001.01453.x

40. Tsukahara H, Shibata R, Ohta N, Sato S, Hiraoka M, Ito S, et al. High levels of urinary pentosidine, an advanced glycation end product, in children with acute exacerbation of atopic dermatitis: relationship with oxidative stress. Metabolism (2003) 52(12):1601-5. doi: 10.1016/S0026-0495(03)00310-X

41. Bao L, Zhang H, Chan LS. The involvement of the JAK-STAT signaling pathway in chronic inflammatory skin disease atopic dermatitis. JAKSTAT (2013) 2(3):e24137-7. doi: 10.4161/jkst.24137

42. Amano W, Nakajima S, Kunugi H, Numata Y, Kitoh A, Egawa G, et al. The Janus kinase inhibitor JTE-052 improves skin barrier function through suppressing signal transducer and activator of transcription 3 signaling. $J$ Allergy Clin Immunol (2015) 136(3):667-677.e667. doi: 10.1016/ j.jaci.2015.03.051

43. Makhmalzade BS, Chavoshy F. Polymeric micelles as cutaneous drug delivery system in normal skin and dermatological disorders. J Adv Pharm Technol Res (2018) 9(1):2-8. doi: 10.4103/japtr.JAPTR_314_17

44. Lennikov A, Kitaichi N, Fukase R, Murata M, Noda K, Ando R, et al. Amelioration of ultraviolet-induced photokeratitis in mice treated with astaxanthin eye drops. Mol Vis (2012) 18:455-64.

45. Park JH, Yeo IJ, Han JH, Suh JW, Lee HP, Hong JT. Anti-inflammatory effect of astaxanthin in phthalic anhydride-induced atopic dermatitis animal model. Exp Dermatol (2018) 27(4):378-85. doi: 10.1111/exd.13437

46. Prausnitz MR, Langer R. Transdermal drug delivery. Nat Biotechnol (2008) 26 (11):1261-8. doi: 10.1038/nbt.1504

47. El Maghraby GM, Barry BW, Williams AC. Liposomes and skin: From drug delivery to model membranes. Eur J Pharm Sci (2008) 34(4):203-22. doi: 10.1016/j.ejps.2008.05.002

48. Jung SH, Cho YS, Jun SS, Koo JS, Cheon HG, Shin BC. Topical application of liposomal cobalamin hydrogel for atopic dermatitis therapy. Die Pharmazie (2011) 66(6):430-5. doi: 10.1691/ph.2011.0829

49. Korting HC, Zienicke H, Schäfer-Korting M, Braun-Falco O. Liposome encapsulation improves efficacy of betamethasone dipropionate in atopic eczema but not in psoriasis vulgaris. Eur J Clin Pharmacol (1990) 39 (4):349-51. doi: 10.1007/BF00315408

Conflict of Interest: The authors declare that the research was conducted in the absence of any commercial or financial relationships that could be construed as a potential conflict of interest.

Copyright (C) 2020 Lee, Jeon, Ham, Lee, Song and Hong. This is an open-access article distributed under the terms of the Creative Commons Attribution License (CC BY). The use, distribution or reproduction in other forums is permitted, provided the original author(s) and the copyright owner(s) are credited and that the original publication in this journal is cited, in accordance with accepted academic practice. No use, distribution or reproduction is permitted which does not comply with these terms. 